\title{
An appreciation of "Cohort analysis of mortality rates as an historical or narrative technique" (RAM Case) $^{1}$
}

\author{
Clive Osmond
}

This might well have been the first epidemiological paper that I ever read. On arrival at the newly formed Medical Research Council Unit in 1980, Donald Acheson and Martin Gardner set me the task of assessing the sitespecific trends in cancer mortality in England and Wales. A widely circulated report was suggesting steep rises in American cancer rates. The authors attributed this to increased occupational exposure to chemicals, though it seemed more likely that it was a consequence of using crude death rates. Was there any evidence of increasing rates in our data? Case's paper was one to which I was directed for ideas on methodology.

When the project was completed, I presented the results and methods at the plenary session of the 1982 Society for Social Medicine scientific meeting in Aberdeen. My co-author, Martin Gardner, happened to be sitting immediately in front of Robert Case. Half way through, Case turned to his neighbour and explained that he had done all this 25 years earlier! No doubt this was a reference to the paper before us.

To what extent was that true? The strength of Case's paper is its articulation of the three dimensions that might influence mortality rates - age, period (year of death), and cohort (generation, year of birth). To the modern statistician, it is difficult to hold back when reading the exposition. All the time the temptation is to interrupt and say, "Yes, you have set out an elegant conceptual basis for analysis, and I have some really appropriate models for exploring it." For the class of log-rates models with Poisson error structure seems tailor made for estimating the importance of the dimensions Case describes. Just define three factored variables in age, period and cohort, go to GLIM or your favourite package, and Bob (Robert Case?) is your uncle. Or so it would seem. ...

Case's paper was clearly written in the dark ages of computation. Surely that is why his contemporaries were so attracted to the simple models of Gompertz and Makeham to describe the age curve. The small number of parameters meant that they could be handled without computers. Nowadays the class of generalised linear models can be fitted to data quite simply. But it is the mathematical formulation which helps to bring out the inherent non-identifiability of the model, and hence the impossibility of the task of apportioning trends uniquely either to period or cohort. This is a problem that is not just a feature of grouped data (see Osmond and Gardner ${ }^{2}$; and note the error in Case's Figure 2, where the $n^{*} n$ cells should really correspond to $2 n$ parallelograms and not $2 n-1$ squares) but is a consequence of the unbreakable rule that "time of birth" + "age at death" = "time at death". Few of us get near enough to travelling at the speed of light to upset that relation! Some progress could be made if it were ever possible to characterise the "biological and inescapable" age curve to which Case refers. However, his own examples show that the age curve may be very different according to whether we are looking cross sectionally or along the cohort.

I will mention three useful adaptations to the basic log-linear model. Firstly, in two expository papers Clayton and Schifflers introduced the concept of drift. ${ }^{34}$ This is the estimable linear trend in rates, which cannot be apportioned either to period or to cohort. An age drift model can be viewed equally as a submodel of age period or age cohort. Secondly, Breslow addressed the problem of poor fit in the full age-period-cohort model. ${ }^{5}$ This may arise, not because the model is badly wrong, but because just a few parameters are being required to explain variations in rates that are based upon a large number of events. Breslow shows how to quantify the extent of the unexplained, extra-Poisson variation. Using this information the simpler submodels may be re-evaluated. Some of them may then provide an adequate fit. Thirdly, Berzuini and Clayton describe a Bayesian approach to the non-parametric smoothing of the sets of age, period and cohort parameters. ${ }^{6}$

These modelling exercises help to summarise, to provide estimates of the precision of parameters, and to project into the future, but they always need to be carried out in the context of the graphical inspection that Case uses. The graphs may well suggest models other than the conventional age-period-cohort model. In Osmond $^{7}$ I show graphs which suggest that coronary heart disease mortality rates in England and Wales have cohort effects which are 
stronger at younger ages. This leads to a mixed model for the rates of the form age* (period + cohort). The new model provides a substantially better fit to the data than the usual age $*$ period* cohort version, and is just identifiable.

This last observation brings me back to Case. His paper contains a fascinating discussion of the possibility that adult disease rates are determined early in life. The focus of my personal research in recent years (see for example Barker ${ }^{8}$ ) has been to attempt to quantify such effects. Coronary heart disease has been the principal example of this "biological programming". Hence my interest in the cohort mortality trends. So this paper has been in- fluential on both my first and latest research. It has been a pleasure to read it again.

1 Case RAM. Cohort analysis of mortality rates as an historical or narrative technique.Br 7 Prev Soc Med 1956;10:159-71. 2 Osmond C, Gardner MJ. Age, period, cohort models. Nonoverlapping cohorts don't resolve the identification problem. Am F Epidemiol 1989;129:31-5.

3 Clayton DG, Schifflers E. Models for temporal variation in cancer rates. I: age-period and age-cohort models. Stat Med 1987;6:449-67.

4 Clayton DG, Schifflers E. Models for temporal variation in cancer rates. II: age-period-cohort models. Stat Med 1987; 6:469-81.

5 Breslow NE. Extra-Poisson variation in log-linear models. Applied Statistics 1984;33:38-44.

6 Berzuini C Clayton D Bayesian analysis of survival on multiple time scales. Stat Med 1994;13:823-38.

7 Osmond C Coronary heart disease mortality trends in Eng land and Wales, 1952-1991. ₹ Public Health Med 1995;17: 404-10.

8 Barker DJP. Mothers, babies, and disease in later life. London BMJ Publishing, 1994. 